\title{
Femtosecond Time-Resolved Wave Packet Motion in Molecular Multiphoton Ionization and Fragmentation
}

\author{
T. Baumert, B. Bühler, M. Grosser, R. Thalweiser, V. Weiss, E. Wiedenmann, \\ Fakultät für Physik, Universität Freiburg, W-7800 Freiburg, Germany \\ and G. Gerber* \\ Fakultät für Physik, Universität Freiburg, W-7800 Freiburg, Germany, and Joint Institute for Laboratory \\ Astrophysics, University of Colorado and National Institute of Standards and Technology, \\ Boulder, Colorado 80309-0440 (Received: February 12, 1991)
}

\begin{abstract}
The dynamics of molecular multiphoton ionization and fragmentation of a diatomic molecule $\left(\mathrm{Na}_{2}\right)$ have been studied in molecular beam experiments. Femtosecond laser pulses from an amplified colliding-pulse mode-locked (CPM) ring dye laser are employed to induce and probe the molecular transitions. The final continuum states are analyzed by photoelectron spectroscopy, by ion mass spectrometry and by measuring the kinetic energy of the formed ionic fragments. Pump-probe spectra employing 70-fs laser pulses have been measured to study the time dependence of molecular multiphoton ionization and fragmentation. The oscillatory structure of the transient spectra showing the dynamics on the femtosecond time scale can best be understood in terms of the motion of wave packets in bound molecular potentials. The transient $\mathrm{Na}_{2}{ }^{+}$ionization and the transient $\mathrm{Na}^{+}$fragmentation spectra show that contributions from direct photoionization of a singly excited electronic state and from excitation and autoionization of a bound doubly excited molecular state determine the time evolution of molecular multiphoton ionization.
\end{abstract}

\section{Introduction}

Multiphoton ionization of diatomic molecules has been studied in recent years by a variety of techniques and is generally well understood. ${ }^{1}$ The ionization is predominantly due to resonance-enhanced multiphoton ionization (REMPI) processes, whereas nonresonant multiphoton processes only play a minor role. Dynamical aspects of the interaction of laser radiation with molecules have been studied by several groups using conventional and zero kinetic energy (ZEKE) photoelectron spectroscopy ${ }^{2}$ as well as ion detection techniques. However, detailed studies of the excitation processes and the different decay channels of highly excited states, which are embedded in the ionization and in the fragmentation continuum, are very rare up to now. The under-

\footnotetext{
* JILA Visiting Fellow 1990-91, University of Colorado, Boulder.
}

standing of the coupling of the different continua of ionization and fragmentation is also of great theoretical interest because of the need for a common treatment of Rydberg states, dissociating states, and doubly excited electronic states to describe the dynamics of highly excited molecular systems. The study of the dissociation and autoionization of $\mathrm{H}_{2}$ via unbound doubly excited states has recently been reported. ${ }^{3}$ Rotational and vibrational autoionization

(1) Multiphoton processes; Smith, S. J., Knight, P. L., Eds.; Cambridge Univ. Press: Oxford, U.K., 1988.

(2) Pratt, S. T.; Dehmer, P. M.; Dehmer, J. L. J. Chem. Phys. 1987, 86, 1727. Allendorf, S. W.; Leahy, D. J.; Jacobs, D. C.; Zare, R. N. J. Chem. Phys. 1989, 91, 2216. Sander, M.; Chewter, L. A.; Muller-Dethlefs, K.; Schlag, E. W. Phys. Rev. A. 1987, 36, 4543.

(3) Verschur, J. W.; van Linden van den Heuvell, H. B. Chem. Phys. 1989, 129, 1. Chupka, W. A. J. Chem. Phys. 1987, 87, 1488. Dixit, S. N.; Lynch, D. L.; McKoy, B. V.; Hazi, A. U. Phys. Rev. 1989, A40, 1700. 
studies of molecular Rydberg states of $\mathrm{H}_{2}, \mathrm{Li}_{2}$, and $\mathrm{Na}_{2}$ have shown how complex direct photoionization can be. ${ }^{4}$ Only recently we have reported on the interaction of a particular bound doubly excited molecular state with different continua and the competition between the various decay channels. ${ }^{5}$ In that study we used femtosecond laser pulses as an experimental tool to distinguish between the dissociative ionization of the molecule and the neutral fragmentation with subsequent excited-fragment photoionization. Both processes, which may lead to formation of the same fragment ion, are difficult to distinguish when using nanosecond or even picosecond laser pulses. This distinction is of particular importance in multiphoton ionization studies of metal cluster systems.

The multiphoton ionization and fragmentation of alkali-metal dimers and, in particular, of $\mathrm{Na}_{2}$ has attracted considerable current interest. In many experiments it has been found that, in conjunction with the formation of $\mathrm{Na}_{2}^{+}$ions, the atomic ions $\mathrm{Na}^{+}$are also formed. REMPI processes via $A^{1} \Sigma_{u}^{+}$or the $B^{1} \Pi_{u}$ states are responsible for this observation. ${ }^{6}$ Ionization and fragmentation studies of $\mathrm{Na}_{2}$ Rydberg states involving vibrational-rotational autoionization have also been reported recently. ${ }^{7}$ However, the final continuum states have been analyzed only in very few experiments. Examples of such detailed studies include the twophoton ionization and dissociation of $\mathrm{Na}_{2}$ resonantly enhanced by the intermediate $2^{1} \Sigma_{u}^{+}$double-minimum state where the kinetic energy of the ionic fragments is measured ${ }^{8}$ and the fragmentation of the neutral molecule $\mathrm{Na}_{2}{ }^{* *}$ into $\mathrm{Na}^{*}(3 \mathrm{p})+\mathrm{Na}^{*}(3 \mathrm{p})$ where Doppler spectroscopy is applied to measure the angular and energy distributions of the neutral fragments.

Time-resolved measurements often open up new directions and provide a more comprehensive view of the physical and chemical processes. Due to recent developments in the generation and amplification of ultrashort light pulses, direct measurements of transient ionization and fragmentation spectra with femtosecond resolution are now possible. This allows a closer look at the dynamical aspects of molecular multiphoton ionization.

In a series of beautiful experiments in the gas phase and in molecular beams, Zewail and co-workers ${ }^{10}$ have demonstrated the enormous advantage of applying femtosecond lasers to the study of molecular dynamics. Their pioneering work in the field of femtosecond photochemistry and transient molecular fluorescence spectroscopy has initiated several other time-resolved ultrafast laser studies. ${ }^{11}$ The observation of transient molecular fluorescence spectra in Zewail's femtosecond pump-probe experiments and the analysis using Heller's wave packet method ${ }^{12}$ show that detailed information about different processes is obtained from the real-time motion of wave packets in potential energy surfaces. Therefore, by probing ultrafast molecular dynamics with femtosecond lasers, the transient characteristics of processes like molecular ionization and fragmentation can also be studied in great detail.

(4) O'Halloran, M. A.; et al. Chem. Phys. Lett. 1988, 146, 291. Mahon, C. R.; Janik, G. R.; Gallagher, T. F. Phys. Rev. 1990, A4I, 3746. Bordas, C.; Labastie, P.; Chevaleyre, J.; Broyer, M. Chem. Phys. 1989, 129, 21. (5) Baumert, T.; Bühler, B.; Thalweiser, R.; Gerber, G. Phys. Rev. Lett. $1990,64,733$.

(6) Keller, J.; Weiner, J. Phys. Rev. 1984, A30, 213. Burkhardt, C. E.; Garver, W. P.; Leventhal, J. J. Phys. Rev. 1985, A3I, 505. Roussell, F.; Breger, P.; Spiess, G. J. Phys. B: At. Mol. Phys. 1985, 18, 3769.

(7) Labastie, P.; Tribollet, B.; Broyer, M.; Bordas, M. C.; Chevaleyre, J. Mol. Phys. 1986, 59, 29. Haugstätter, R.; Goerke, A.; Hertel, I. V. Phys. Rev. 1989, A39, 5085. Bordas, C.; Labastie, P.; Brevet, P. F.; Broyer, M. Phys. Rev. 1989, A40, 1696.

(8) Haugstātter, R.; Goerke, A.; Hertel, I. V. Z. Phys. 1988, D9, 153 (9) Gerber, G.; Möller, R. Phys. Rev. Lett. 1985, 55, 814. Photons and Continuum States of Atoms and Molecules; Rahman, N. K., Guidotti, C., Allegrini, M., Eds.: Springer Proceedings in Physics; Springer Verlag: Berlin, 1987; Vol. 16, p 126.

(10) Rosker, M.: Dantus, M.; Zewail, A. J. Chem. Phys. 1988, 89, 6113, and references therein. Bowman, R. M.; Dantus, M.; Zewail, A. Chem. Phys. Lett. 1989, 161, 297; 1990, 174, 546. Grübele, M.; et al. Chem. Phys. Lett. $1990,166,459$.

(11) Glownia, J. H.; Misewich, J. A.; Sorokin, P. P. J. Chem. Phys. 1990 92, 3335 . Scherer, N. F.; Ruggiero, A. J.; Du, M.; Fleming, G. R. J. Chem. Phys. 1990, 93, 856. Baumert, T.; Bühler, B.; Thalweiser, R.; Gerber, G. Phys. Rev. Lett. 1990, 64, 733.

(12) Heller, E. J. Acc. Chem. Res. 1981, 14, 368.

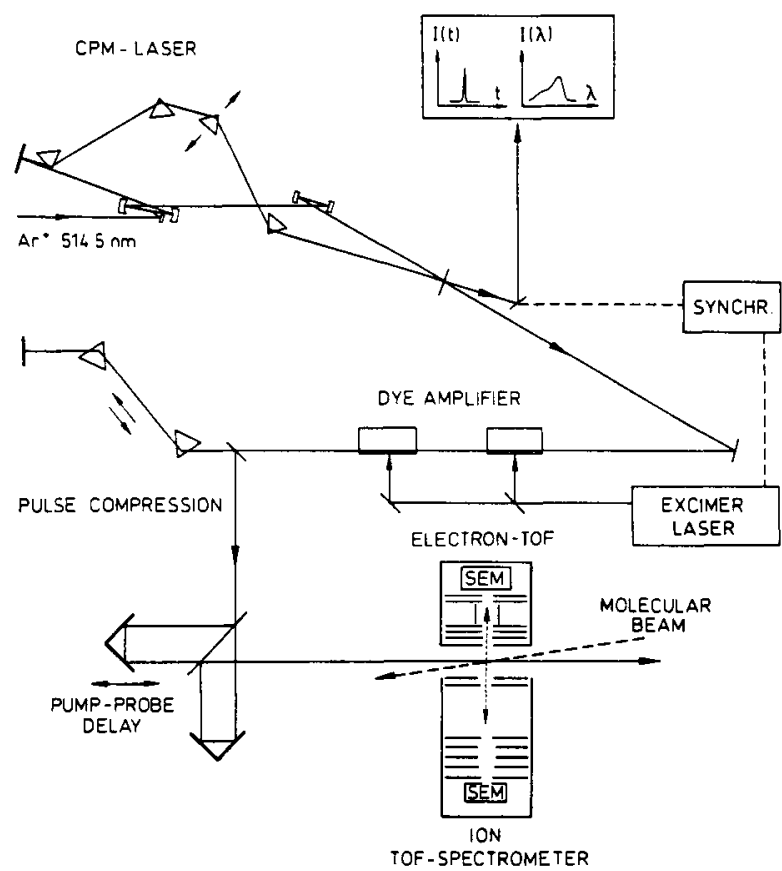

Figure 1. Femtosecond pump-probe laser and molecular beam arrangement. Pump and probe laser beams are collinear and perpendicular to the molecular beam and the electron and ion TOF spectrometers.

In this paper we present and discuss experimental results of time-resolved studies of molecular multiphoton ionization of the diatomic sodium molecule in molecular beam experiments applying femtosecond pump-probe techniques and ion and electron spectroscopy.

\section{Experiment}

In our femtosecond laser-molecular beam studies of multiphoton ionization processes, we have used a combination of experimental techniques. Femtosecond laser pulses are used to induce and probe the molecular transitions. A supersonic molecular beam provides the $\mathrm{Na}_{2}$ molecules in a collision-free environment and restricts the set of initial states to $v^{\prime \prime}=0, J^{\prime \prime}$. Time-of-flight (TOF) spectroscopy is used to determine the masses of the ions, the released kinetic energy of the ionic fragments, and the energy distribution of the ejected electrons. Because the ion and electron collection angles are fixed, the ion and electron angular distributions can be studied by rotating the laser polarization.

The laser system and the schematic experimental arrangement of the molecular beam and the ion and electron TOF spectrometers are shown in Figure 1. A supersonic sodium beam is produced either by a pure sodium expansion through a small orifice of typically $0.15-\mathrm{mm}$ diameter or by an expansion seeded with argon. The oven is usually operated at $1000 \mathrm{~K}$ with nozzle temperatures about $50 \mathrm{~K}$ higher. Due to the cooling effect of the supersonic expansion only the vibrational level $v^{\prime \prime}=0$ in the $\mathrm{X}^{1} \Sigma_{\mathrm{g}}^{+}$ground state of $\mathrm{Na}_{2}$ is sufficiently populated. The laser-molecular beam interaction region is placed between parallel plates so that the formed ions and electrons can be detected in opposite directions by two differently designed TOF spectrometers. Both TOF spectrometers are perpendicular to the laser beam and the molecular beam. When the ions are extracted with a low electric field $(5-10 \mathrm{~V} / \mathrm{cm})$, the ion time-of-flight depends on the mass and on the projection of the initial fragment velocity onto the spectrometer axis. Thus, by knowing the extraction field, the measured time-of-flight spectrum is transformed into a velocity spectrum, from which upper bounds of the fragment kinetic energy are determined with an accuracy of about $\pm 500 \mathrm{~cm}^{-1}$. This experimental technique is widely known. ${ }^{13}$ The mass resolution

(13) See, for example, Ogorzalek Loo, R.; Hall, G. E.; Harri, H. P.; Houston, P. L. J. Phys. Chem. 1988, 92, 5. 

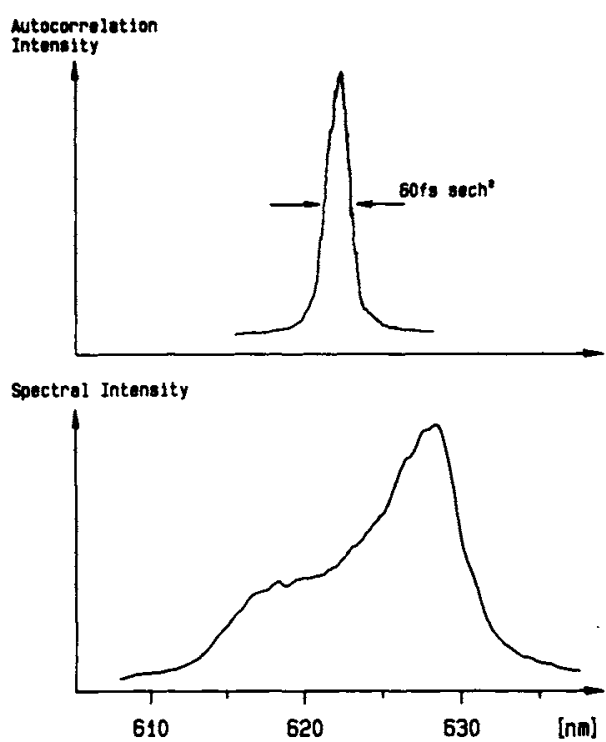

Figure 2. (Top) CPM pulse duration measured by autocorrelation technique. (Bottom) Spectral distribution of the $60-\mathrm{fs}$ laser pulse.

$m / \Delta m$ of our ion TOF spectrometer is about 100 and is sufficient to study molecules and small metal-cluster systems. The energy calibration of the electron TOF spectrometer is based upon several one- and two-photon resonance-enhanced ionization processes in atomic sodium, which produce electrons with a well-defined kinetic energy. The energy resolution of the electron TOF spectrometer is $25-80 \mathrm{meV}$ in the range $0.1-3 \mathrm{eV}$.

Femtosecond laser pulses are generated in a home-built colliding-pulse mode-locked (CPM) ring dye laser following the design of Valdmanis and Fork. ${ }^{14}$ Compensation of the intracavity group velocity dispersion (GVD) with the standard four-prism arrangement produces pulses of 60 -fs duration at the center wavelength of $627 \mathrm{~nm}$ with Rhodamine $6 \mathrm{G}$ as the gain medium. The emission peak of the CPM dye laser can be shifted in the range $612-632 \mathrm{~nm}$ by adjusting the DODCI absorber concentration, resulting however in a slight increase of the output pulse duration. With $4-W$ pump power of $514.5 \mathrm{~nm} \mathrm{CW} \mathrm{Ar}{ }^{+}$laser light, we obtain CPM output pulses with an average power of $15 \mathrm{~mW}$ in a $75-\mathrm{MHz}$ pulse train. The spectral distribution of the output pulses is measured with an optical multichannel analyzer (OMA). The pulse duration is determined by the autocorrelation technique using anticollinear second harmonic generation (SHG) in a $1-\mathrm{mm}$ KDP crystal and assuming a sech ${ }^{2}$ pulse shape. ${ }^{15}$ The spectral distribution and the time duration of the CPM pulses are monitored during the experiment. They are both shown in Figure 2. Since the CPM pulse energies are much too low (100-200 pJ) for our molecular beam experiment, we employed dye amplifiers to increase the pulse energies to a level suited for the chosen experiment. With a two-stage rhodamine 101 dye amplifier, transversely pumped by $3 \mathrm{~mJ} / 5 \mathrm{~mJ}$ of a $308-\mathrm{nm}$ excimer laser (Lambda Physik LPX 120), which is synchronized to the CPM, we obtained output pulses of 5- $\mu \mathrm{J}$ energy. Amplified spontaneous emission (ASE) is efficiently suppressed by spatial filtering after the first and the second stage and by the $10-\mathrm{m}$ distance between the laser and the experiment.

The resulting pulse broadening, mainly due to GVD in the dye amplifiers was reduced in the pulse compression unit. The amplified and recompressed 70-fs laser pulses of $100 \AA$ spectral width are nearly transform limited. The Fourier transform limit of 70-fs laser pulses assuming sech ${ }^{2}$ pulse shapes is $60 \AA$. Experimentally it is difficult to reach that limit because of nonlinear chirp contributions in the amplification process. At an earlier stage of the experiment ${ }^{5}$ we had amplified the CPM output $(150-\mathrm{MHz}$ pulse train) in a one-stage $\mathrm{N}_{2}$-laser-pumped rhodamine $6 \mathrm{G}$ dye amplifier

(14) Valdmanis, J. A.; Fork, R. L. IEEE J. Quantum Electron. 1986, 22, 112.

(15) Ippen, E. P.; Shank, C. V. Ultrafast Light Pulses, Shapiro, S. L., Ed.; Topics in Applied Physics; Springer-Verlag: Berlin, 1977; Vol. 18, p 83.

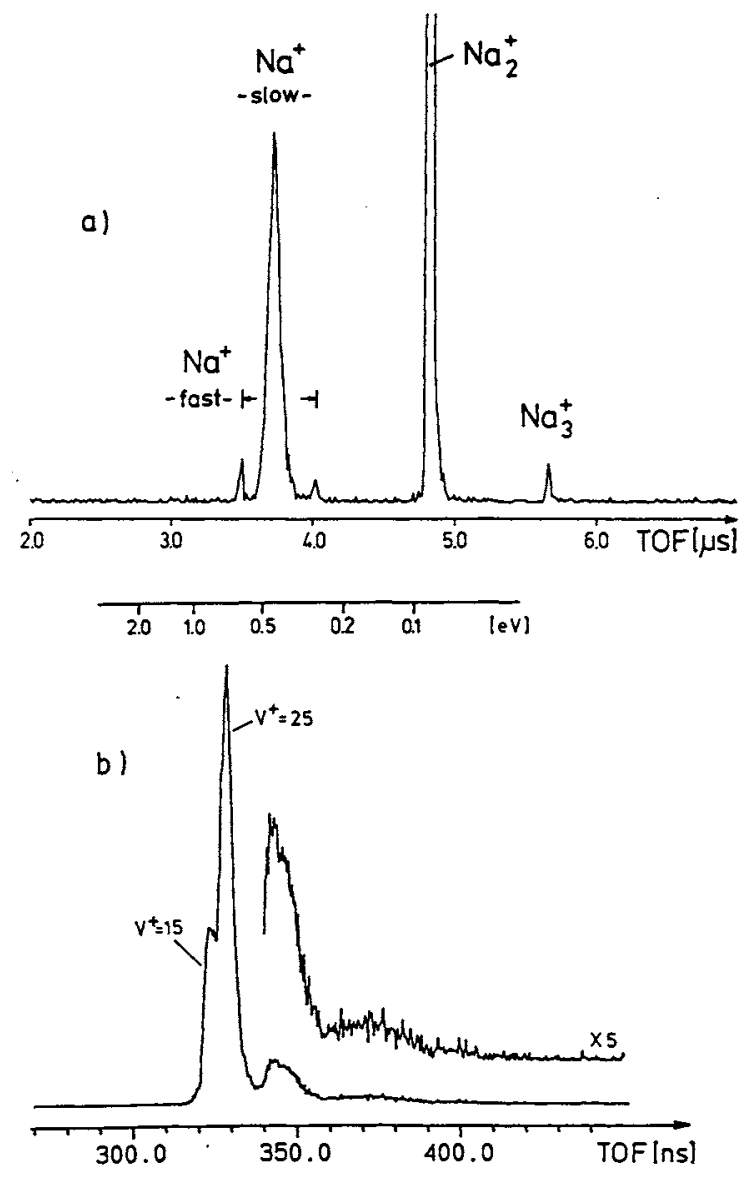

Figure 3. (a) Time-of-flight spectrum of ions formed by the interaction of $120-\mathrm{fs}$ laser pulse of $10-\mathrm{nJ}$ energy with sodium molecular beam. The central wavelength was $616 \mathrm{~nm}$. (b) Time-of-flight spectrum of ejected electrons. The energy scale refers to the electron TOF.

to produce pulses of 10-nJ energy and 120-fs time duration. For the pump-probe experiment we used a Michelson arrangement to delay the probe laser relative to the pump laser. A stepper-motor-driven linear precision actuator with $0.05-\mu \mathrm{m}$ feed in one arm of the interferometer sets the time delay. Both the pump and the probe laser beams enter the interaction region collinearly with the same polarization and perpendicular to the molecular beam. We used recompressed laser pulses of 70-fs duration, about $100-\AA$ spectral width, and $0.2-\mu \mathrm{J}(I \approx 50$ $\mathrm{GW} / \mathrm{cm}^{2}$ ) for both the pump and the probe. The laser pulse energy was kept this low in the pump-probe experiment to simplify the study of the basic physical processes.

\section{Results and Discussion}

The ion TOF spectrum displayed in Figure 3a is obtained when femtosecond laser puises of $120-\mathrm{fs}$ duration and $10-\mathrm{nJ}$ pulse energy $\left(I \approx 100 \mathrm{MW} / \mathrm{cm}^{2}\right)$ interact with the sodium molecular beam. The spectrum clearly shows the observation of $\mathrm{Na}_{3}^{+}, \mathrm{Na}_{2}^{+}$, and "slow" $\mathrm{Na}^{+}$as well as "fast" $\mathrm{Na}^{+}$ionic fragments resulting from the fs laser excitation at $\lambda_{\max }=616 \mathrm{~nm}$. For a seeded sodium expansion, higher laser pulse energy of $5 \mu \mathrm{J}$ and wavelengths around $618 \mathrm{~nm}$, we observed sodium cluster ions $\mathrm{Na}_{n}^{+}$up to $n=$ 8. The small signal of the metal cluster $\mathrm{Na}_{3}^{+}$in Figure $3 \mathrm{a}$ is due to an absorption of two photons of $616 \mathrm{~nm}$, which are sufficient to ionize the $\mathrm{Na}_{3}$ cluster. ${ }^{16}$ The intense $\mathrm{Na}_{2}^{+}$signal is due to a resonance-enhanced three-photon ionization process of $\mathrm{Na}_{2}$, as has been discussed in detail in a recent paper ${ }^{5}$ from our group.

The results of that paper are shortly summarized here. The ionization-enhancing intermediate electronic state $2^{1} \Pi_{8}(3 \mathrm{~s}, 3 \mathrm{~d})$ is populated by a two-photon absorption process. The $2^{1} \Pi_{8}$ state is then photoionized by absorption of a third photon leading to

(16) Broyer, M.; Delacretaz, G.; Labastie, P.; Whetten, R. L.; Wolf, J. P.; Wöste, L. Z. Phys. 1986, D3, 131. 


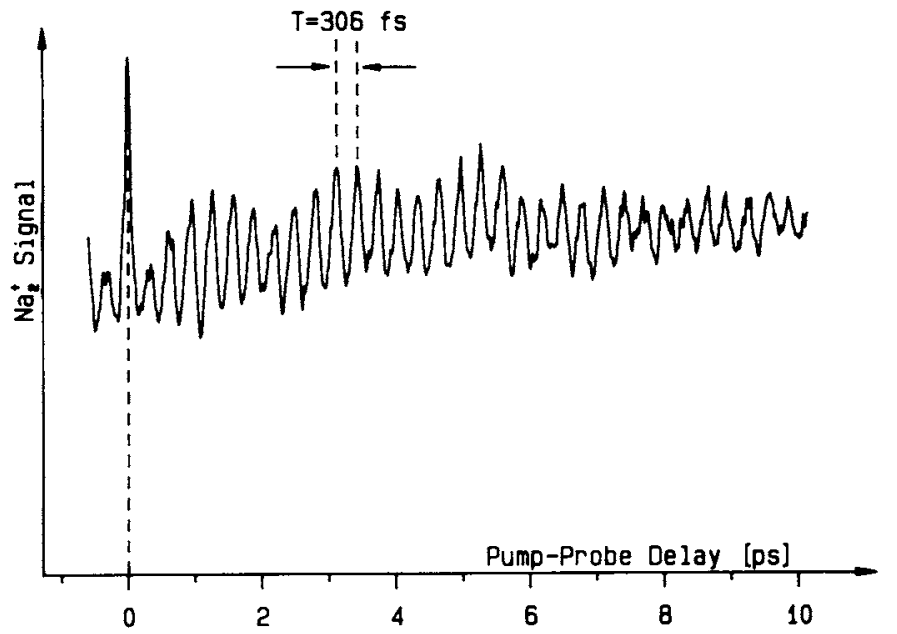

Figure 4. Pump-probe time-delay spectrum of $\mathrm{Na}_{2}^{+}$obtained with 70-fs pump and probe pulses of $0.2-\mu \mathrm{J}$ energy at $\lambda_{\max }=627 \mathrm{~nm}$. The envelope intensity variation and the oscillatory structure of this transient $\mathrm{Na}_{2}^{+}$ multiphoton ionization signal reveal two contributions out of phase by $180^{\circ}$. They correspond to independent wave packet motions in bound molecular potentials with 306- and 363-fs oscillation periods.

the observed strong $\mathrm{Na}_{2}^{+}$signal. In this direct photoionization, the vibrational levels $v^{+}=14,15$ and $v^{+}=24,25$ of the electronic group state $\mathrm{X}^{2} \Sigma_{\mathrm{g}}^{+}$of the dimer ion are preferentially formed as the measured photoelectron spectrum, displayed in Figure $3 \mathrm{~b}$, shows. The observed electrons, which have kinetic energies of $E=810 \pm 10 \mathrm{meV}$ and $E=940 \pm 10 \mathrm{meV}$ even show an intensity ratio that agrees with the calculated Franck-Condon factors.

Considering the 120 -fs time duration of the laser pulses, the observed "slow" and "fast" $\mathrm{Na}^{+}$ionic fragments have to originate from processes occurring at small internuclear distances. Therefore, predissociation of $\mathrm{Na}_{2}{ }^{*}$ and photoionization of $\mathrm{Na}^{*}$ as the origin of the ionic fragments can be ruled out. Absorption of one more photon from still the same femtosecond laser pulse induces the bound-free transition ${ }^{2} \Sigma_{g}^{+}-2 \Sigma_{u}^{+}$in the molecular ion $\mathrm{Na}_{2}^{+}$leading to recoil energies $W$ of the ionic fragments $\mathrm{Na}^{+}$that agree with the value $W=10500 \pm 500 \mathrm{~cm}^{-1}$ obtained from the analysis of the ion TOF spectrum.

On the basis of both the measured electron spectrum and the distribution of recoil energies $W=900 \pm 400 \mathrm{~cm}^{-1}$ obtained from the analysis of the "slow" ionic fragments $\mathrm{Na}^{+}$, the excitation and the decay of doubly excited molecular states of $\mathrm{Na}_{2}$ is found to be a second multiphoton ionization channel for diatomic sodium. The electronic autoionization of a bound state of $\mathrm{Na}_{2}{ }^{* *}$ into the two continua ${ }^{2} \Sigma_{\mathrm{g}}^{+}$and ${ }^{2} \Sigma_{\mathrm{u}}^{+}$as well as the autoionization-induced fragmentation $\mathrm{Na}_{2}^{* *} \rightarrow \mathrm{Na}^{+}+\mathrm{Na}+\mathrm{e}^{-}\left(E_{\mathrm{Kin}}\right)+W$ are very important processes with regard to the dynamics of highly excited molecules.

A very interesting question is, what happens with the molecule during the multiphoton ionization and fragmentation when we look at it on a femtosecond time scale? What are the dynamics of these processes, and does the molecule absorb one photon after another or all at once? Our interest is focused on the dynamics of molecular multiphoton ionization on a femtosecond time scale and on the coherence or incoherence of the contributions to the ionization step.

The first time-resolved studies of molecular multiphoton ionization in a molecular beam using femtosecond pump-probe techniques ${ }^{17}$ revealed unexpected features of the dynamics of the absorption of many photons by a diatomic molecule. The observed femtosecond pump-probe delay spectrum, corresponding to the transient $\mathrm{Na}_{2}^{+}$ionization spectrum is shown in Figure 4 . The experimental parameters of the measurement were $70-\mathrm{fs}$ laser pulse duration, $0.2-\mu \mathrm{J}$ pulse energy, and $\lambda_{\max }=627 \mathrm{~nm}$. The spectrum shows a beat structure superimposed on a strong modulation of

(17) Baumert, T.; Grosser, M.; Thalweiser, R.; Gerber, G. Phys. Rev. Lett., submitted for publication.

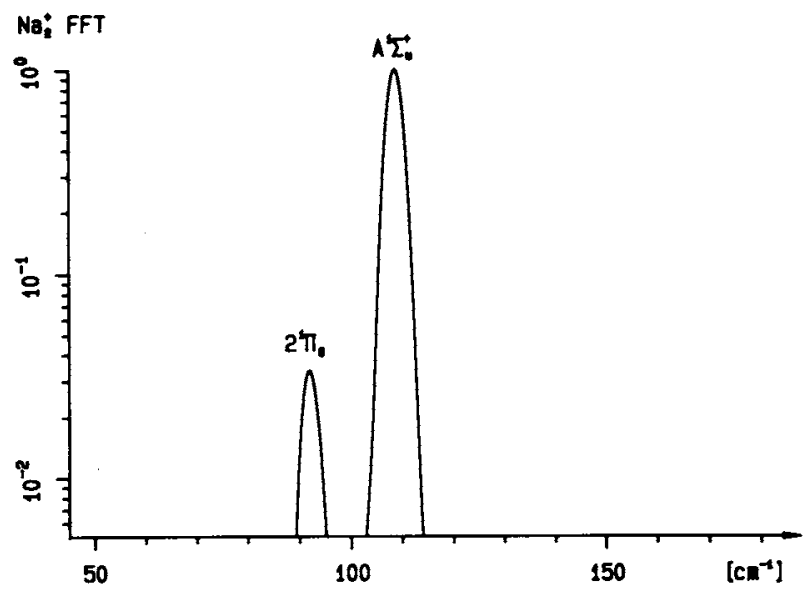

Figure 5. Fast Fourier transform spectrum of transient $\mathrm{Na}_{2}^{+}$ionization spectrum (Figure 4). The two frequency groups are attributed to the vibrational eigenfrequencies of the $\mathrm{A}^{1} \Sigma_{u}^{+}$state $\left(v^{\prime}=10-14\right)$ and the $2^{1} \Pi_{8}$ state $\left(v^{*}=11-18\right)$.

the ionization signal. Because pump and probe are identical the signal is symmetric around zero time delay. The modulation period estimated from the peak-to-peak separation is $T=306 \mathrm{fs}$. Evident from the beat structure in Figure 4, there are two major contributions to the transient ionization spectrum, and the envelope intensity variation reveals them to be about $180^{\circ}$ out of phase.

This is easy to understand from the superposition of two oscillations. For two oscillations in phase at zero delay time the envelope intensity is at its maximum value for $\Delta t=0$. However, the superposition of two oscillations with a phase shift of $180^{\circ}$ at $\Delta t=0$ leads to an envelope variation showing its minimum value at $\Delta t=0$ and increasing values for increasing delay times $\Delta t$.

The strong modulation of the signal decreases for longer delay times. With the given experimental parameters, the observed dynamics can best be understood in terms of the motion of wave packets in bound molecular potentials.

A Fourier analysis of the pump-probe delay spectrum (Figure 4 ) is performed and the result is displayed in Figure 5. The power spectrum density shows two major groups of frequencies, one centered at $108.7 \mathrm{~cm}^{-1}$ in the interval from 106.9 to $110.5 \mathrm{~cm}^{-1}$, and a second centered at $92.0 \mathrm{~cm}^{-1}$ in the interval from 90.2 to $93.9 \mathrm{~cm}^{-1}$. The individual frequencies are unresolved due to the scan length of the pump-probe spectrum, since the FFT resolution is proportional to $1 / \Delta t_{\max }$. For higher laser intensity and $\lambda_{\max }$ $=618 \mathrm{~nm}$, additional lower frequency components appear, which are attributed to wave packet motion in the $4^{1} \Sigma_{\mathrm{g}}^{+}$shelf state and will be discussed elsewhere. ${ }^{18}$

From the transient $\mathrm{Na}_{2}^{+}$ionization spectrum (Figure 4) and the Fourier frequencies, we identify two major contributions to the multiphoton ionization of $\mathrm{Na}_{2}$. First we shall focus on the discussion of the "in phase" wave packet motion $(T=306 \mathrm{fs})$. The $\mathrm{Na}_{2}$ molecules in the ground state $\mathrm{X}^{1} \Sigma_{\mathrm{g}}^{+}$and $v^{\prime \prime}=0$ are pumped into excited electronic states by a laser pulse whose 70 -fs duration is much shorter than the vibrational period of $\mathrm{Na}_{2}$. The classical vibrational period-as given by $T($ vib) $=1 / v(E)$, where $v(E)$ is the local vibrational frequency-for the $v^{\prime}=11,12$ states of the excited electronic A state is just $306.5 \mathrm{fs}$. Since the motion of a wave packet in a bound molecular potential is uniquely determined by the vibrational level spacings, the observed short time oscillation of $T=306 \mathrm{fs}$ is directly related to the $\mathrm{A}^{1} \Sigma_{\mathrm{u}}^{+}$state. Thus, the spectrally broad pump laser forms a coherent superposition of the vibrational eigenstates $v^{\prime}=10-14$ in the excited A state. Because a coherent superposition of an increasing number of vibrational eigenstates approaches the classical limit of the heavy-particle motion, the vibrational wave packet formed in such a way will oscillate between the classical turning points of the A-state potential well. Since wave packets are well localized in

(18) Baumert, T.; Weiss, V.; Gerber, G. To be published. 

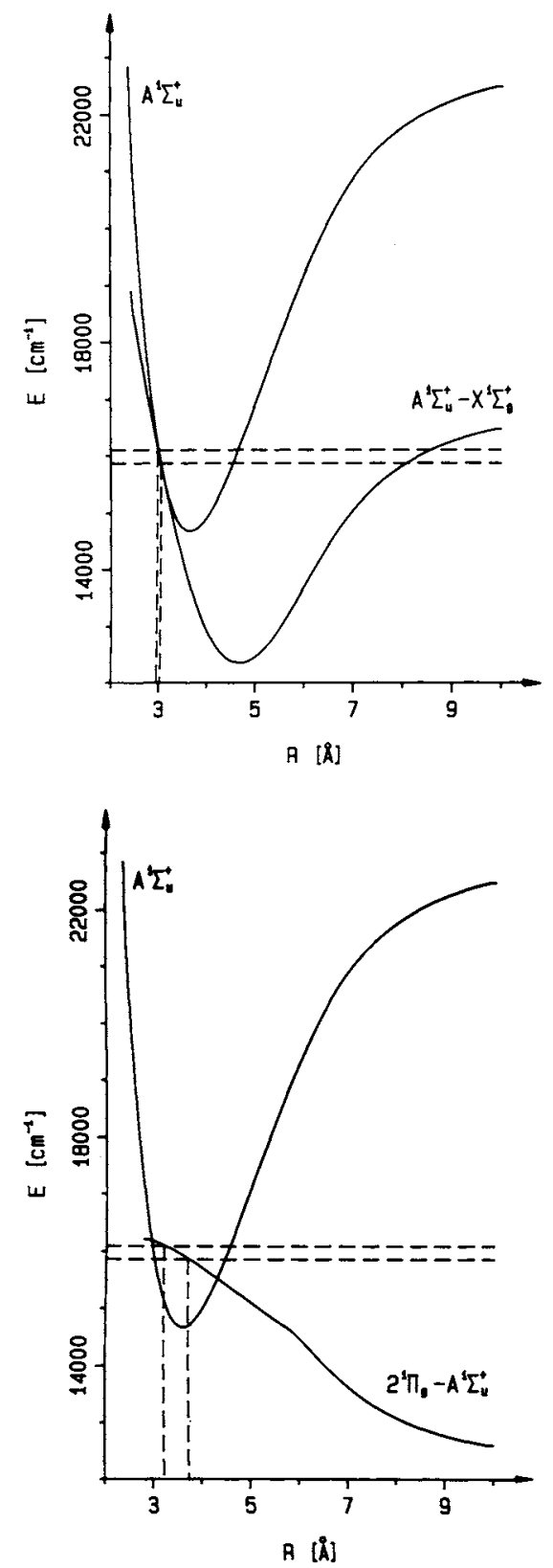

Figure 6. (a, Top) Intersection of A-state potential curve with the difference potential between the $A^{1} \Sigma_{u}^{+}$state and the $X^{1} \Sigma_{g}^{+}$state of $\mathrm{Na}_{2}$ at the inner turning point. For the given pump pulse frequencies the coherent excitation of the $v^{\prime}$ levels occurs within a very narrow range of internuclear distances. (b, Bottom) Intersection of the $2^{1} \Pi_{\mathrm{g}}-\mathrm{A}^{1} \Sigma_{u}^{+}$difference potential with the A-state potential curve. This shows that for the given probe pulse energies transitions from the $A$ state fo the $2^{1} \Pi_{8}$ state can only occur within a restricted range of internuclear distances.

space and their time evolution is the quantum-mechanical counterpart to the classical motion of the nuclei, classical concepts like Mulliken's difference potential ${ }^{19}$ are applicable to the interpretation of the results. The motion of this wave packet is determined by the vibrational energy levels of the A state, ${ }^{20}$ which are $109.5,108.8,108.1$, and $107.4 \mathrm{~cm}^{-1}$ for the levels $v^{\prime}=10-14$. These values agree with the frequency components derived from the Fourier analysis. The shape of the wave packet depends on the amplitudes of the components, which are determined by the pump laser spectral distribution, the levels $v^{\prime \prime}$ in the $\mathrm{X}$ ground state, and the Franck-Condon factors of the involved transitions. Since in our sodium molecular beam the only populated lower level is $v^{\prime \prime}=0$, the vibrational wave packet in the $\mathrm{A}^{1} \Sigma_{\mathrm{u}}^{+}$state is formed

(19) Mulliken, R. S. J. Chem. Phys. 1971, 55, 309.

(20) Gerber, G.; Möller, R. Chem. Phys. Lett. 1985, 113, 546. Knöckel, H.; Richter, H.; Johr, F.; Tiemann, E. Chem. Phys. 1991, 152, 399.

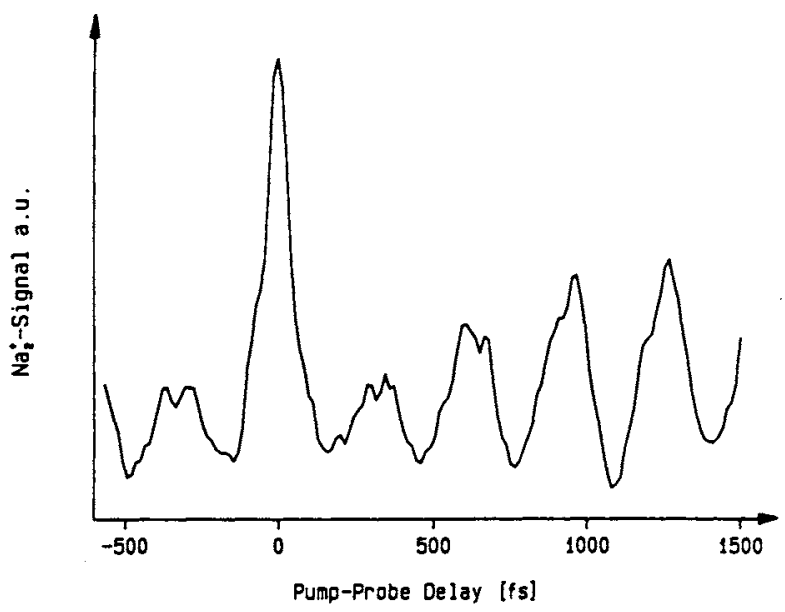

Figure 7. Enlarged portion of $\mathrm{Na}_{2}^{+}$transient ionization spectrum showing an unresolved doublet structure for each peak.

right at the inner turning points. This is illustrated in Figure 6a, where the $A-X$ difference potential curve intersecting the $A$ state at $v^{\prime}=10-14$, shows that transitions from $v^{\prime \prime}=0$ to these upper levels occur, within the pump laser bandwidth, only at the inner turning points within a very narrow range of internuclear distances. The motion of the wave packet in the A-state potential is probed after a time delay $\Delta t$ by a 70 -fs probe pulse and is transferred via the $2^{1} \Pi_{g}(3 s, 3 \mathrm{~d})$ Rydberg state into the $\mathrm{Na}_{2}^{+}\left(\mathrm{X}^{2} \Sigma_{\mathrm{g}}^{+}\right)$ionization continuum. From the oscillatory $\mathrm{Na}_{2}^{+}$signal (period $306 \mathrm{fs}$ ), which is in phase with the preparation of the wave packet at the inner turning point at time $t=0$, the motion of the wave packet in the A state is evidently probed only near the inner turning point. Probing at the outer turning point would result in a $180^{\circ}$ phase shift, which, however, is not observed with the $306 \mathrm{fs}$ motion. Note that a direct two-photon transition from the $A$ state into the $\mathrm{Na}_{2}^{+}\left(\mathrm{X}^{2} \Sigma_{\mathrm{g}}^{+}\right)$ionization continuum results in a time-independent ionization signal, because the shapes of the two potentials are so similar. For instance, the Franck-Condon factor for the transition from the neutral $\mathrm{A}^{1} \Sigma_{u}^{+}, v^{\prime}=0$ state to the ground state of the $\mathrm{Na}_{2}^{+}$ ion ${ }^{2} \Sigma_{\mathrm{g}}^{+}, v^{+}=0$ is 0.976 , and 0.906 for the transition $v^{\prime}=20$ to $v^{+}=20$. Thus, ionizing transitions, leading to electron energies of about $7050 \mathrm{~cm}^{-1}$ for the applied laser wavelengths, may occur with equal probability for all internuclear distances between the inner and outer turning points. Analysis based on the difference potentials $2^{1} \Pi_{\mathrm{g}}-\mathrm{A}^{1} \Sigma_{\mathrm{u}}^{+}$(displayed in Figure $6 \mathrm{~b}$ ) and $\mathrm{Na}_{2}^{+}\left({ }^{2} \Sigma_{\mathrm{g}}^{+}\right)-\mathrm{Na}_{2}\left({ }^{1} \Pi_{\mathrm{g}}\right)$ (see Figure $9 \mathrm{~b}$ ) shows that only through the resonant intermediate $2^{1} \Pi_{8}$ state, which acts as a "window" for the two-photon probe transition, can the time-dependent motion of the A-state wave packet be seen. In fact the difference potential in Figure $6 \mathrm{~b}$ shows the $2^{1} \Pi_{\mathrm{g}}$ state restricts the possible transitions to a region close to the inner turning point. Since the ionizing transitions do not occur exactly at the inner turning point, a double structure is expected to be seen in the transient signal. In an enlarged portion of the pump-probe spectrum, displayed in Figure 7 , this double structure is clearly seen.

The relevant RKR potential curves and the preparation and probing of the A-state wave packet motion are shown in Figure 8. As indicated by the strong ionization signal (Figure 4) around zero time delay, the three photons of this one-photon-pump and two-photon-probe direct photoionization process are absorbed instantaneously or at least within the time overlap of the pump and the probe pulses. The two-photon-probe ionizing transition occurs then periodically after each round trip. Note that the oscillation of the transient $\mathrm{Na}_{2}^{+}$ionization signal disappears with increasing pump-probe delay time, as is expected from the dispersion of the wave packet, which is determined by the anharmonicity of the potential well.

The Fourier analysis (Figure 5) of the transient $\mathrm{Na}_{2}^{+}$ionization signal and the observed beat structure (Figure 4) imply a second major ionization channel. This ionization channel, which is connected with the "out-of-phase" wave packet motion, will be discussed in the following. On the basis of the derived Fourier 


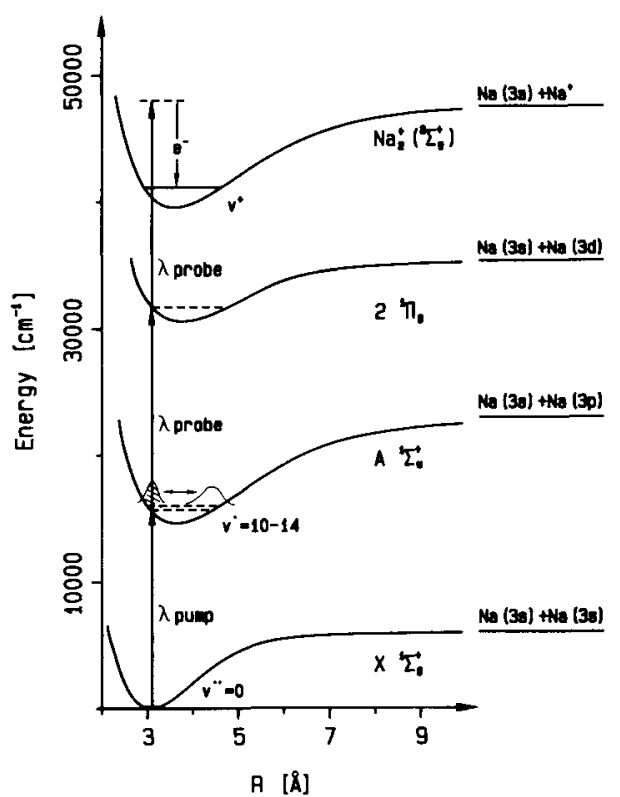

Figure 8. Potential curve diagram illustrating preparation of the wave packet in the A-state at the inner turning point and two-photon probe process transferring motion of the wave packet into the ionization continuum via the $2^{\prime} \Pi_{\mathrm{g}}$ state.

components of about $92 \mathrm{~cm}^{-1}$ and the femtosecond laser wavelengths of about $627 \mathrm{~nm}$, we conclude that in this second channel the pump laser creates a coherent superposition of vibrational levels in the $2^{1} \Pi_{\mathrm{g}}$ state by a two-photon transition. The absorption of two laser photons induces transitions from $\mathrm{X}^{1} \Sigma_{\mathrm{g}}^{+}, v^{\prime \prime}=0$ to vibrational levels $v^{*}=11-18$ of the $2^{1} \Pi_{\mathrm{g}}$ state. By use of the known spectroscopic constants of the $2^{1} \Pi_{\mathrm{g}}$ state, ${ }^{21}$ the vibrational spacings of these coherently excited levels are calculated. They range from 89.7 to $94.1 \mathrm{~cm}^{-1}$ and agree with the frequencies $90.2-93.9 \mathrm{~cm}^{-1}$ obtained in the Fourier analysis. The vibrational wave packet in the $2^{1} \Pi_{8}$ state is also formed definitely at the inner turning point of that state, as the difference potential $2^{1} \Pi_{\mathrm{B}}-\mathrm{X}^{\mathrm{I}} \Sigma_{\mathrm{B}}^{+}$, displayed in Figure 9a, and calculations of Franck-Condon accumulation integrals for the coherently excited levels $v^{*}=11-18$ show. With a time-delayed femtosecond probe pulse, the real-time motion of the wave packet in the $2^{1} \Pi_{\mathrm{g}}$ potential well is detected. A onephoton probe process transfers the motion of this wave packet into the ionization continuum, but only, as the phase shift of $180^{\circ}$ clearly shows, at the outer turning point. Hence, this is a multiphoton ionization process where first two pump photons are absorbed at the inner turning point and then, at the earliest time after half of the $v^{*}$ vibrational period of about $175-185 \mathrm{fs}$, the probe photon is absorbed at the outer turning point. The probe transition again occurs periodically, but now at the outer turning point. Definitely, this is a sequential multiphoton ionization process where first at $t=0$ the two pump photons are absorbed and then about $t=175-185$ fs later the probe-photon-induced transition takes place.

An interesting question is now, are these two contributions to the observed oscillating $\mathrm{Na}_{2}^{+}$ionization signal just two different intramolecular ionization pathways, whose amplitudes have to be added coherently to account for the observation, or do these contributions come from two independent multiphoton ionization processes resulting in distinguishable final states, to require incoherent addition of intensities?

If we apply only Franck-Condon arguments, there is no reason why direct photoionization of the Rydberg electron should take place only at the outer turning point for the given vibrational levels $v^{*}$. Moreover, the difference potential analysis applied to the probe transition $\mathrm{Na}_{2}^{+}\left(2^{1} \Pi_{\mathrm{g}}, v^{*}=11-18\right) \rightarrow \mathrm{Na}_{2}^{+}\left({ }^{2} \Sigma_{\mathrm{g}}^{+}, v^{+}\right)$and also the recently performed wave packet calculations by Engel $^{22}$ show that

(21) Taylor, A. J.; Jones, K. M.; Schalow, A. L. J. Opt. Soc. Am. 1983, 73, 994. Whang, T. J.; Wang, H.; Lyyra,LiLi, A. M.; Stwalley, W. C. J. Mol. Spectrosc. 1991, 145, 112.
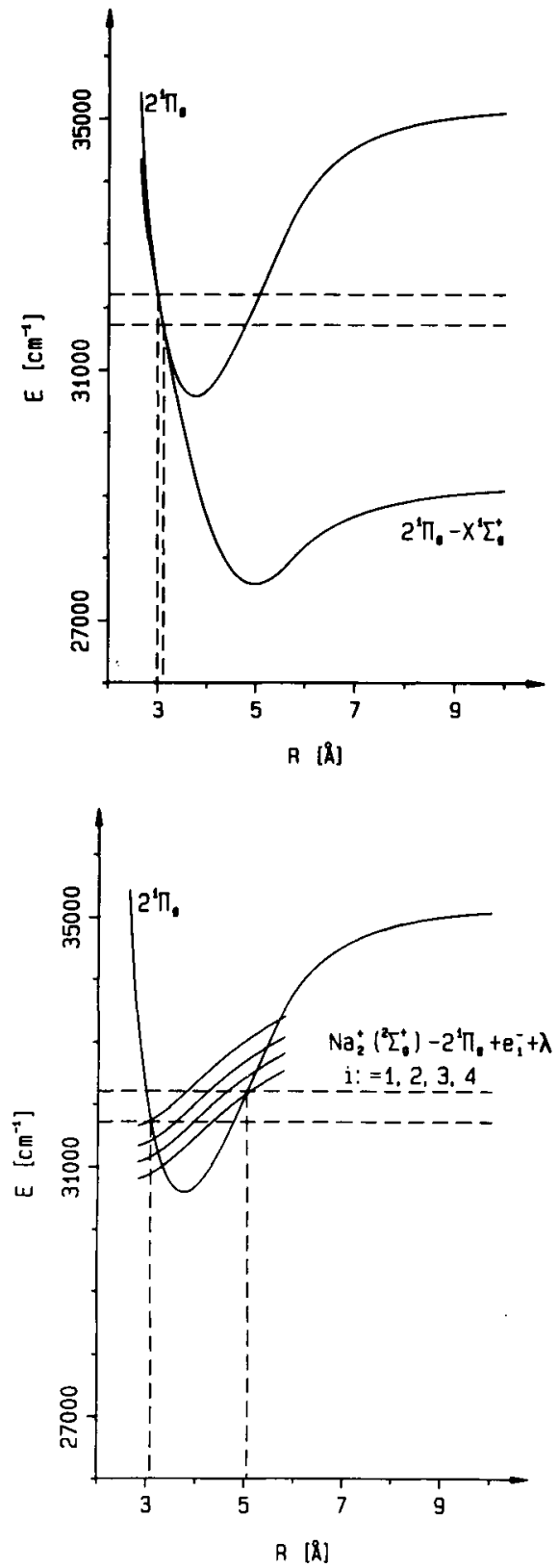

Figure 9. (a, Top) Intersection of the $2^{1} \Pi_{\mathrm{g}}-\mathrm{X}^{1} \Sigma_{\mathrm{g}}^{+}$difference potential with the $2^{1} \Pi_{\beta}$ state potential curve at the inner turning points of coherently excited $v^{*}$ levels. The range of internuclear distances for the two-photon pump transitions $\mathrm{X}^{1} \Sigma_{\mathrm{a}}^{+}-2^{1} \Pi_{\mathrm{g}}$ are also very restricted. (b, Bottom) Difference potential analysis applied to the $\mathrm{Na}_{2}\left(2^{\prime} \Pi_{\mathrm{g}}\right)-\mathrm{Na}_{2}^{+}\left(\mathrm{X}^{2} \Sigma_{\mathrm{g}}^{+}\right)$bound-continuum transition. In this direct photoionization process essentially four different groups of electrons are produced for each $v^{*}$ level. While the range of internuclear distances is different for each group, the summation over all electron energies leads to an $R$-independent ionization signal.

direct photoionization of the $2^{1} \Pi_{g}$ state results in a time-independent contribution to the observed $\mathrm{Na}_{2}^{+}$signal.

The table of Franck-Condon factors calculated for this probe transition shows four diagonals with high transition probabilities leading essentially to four different electron energies for each lower $v^{*}$ vibrational level. The difference potential $\mathrm{Na}_{2}^{+}\left(\mathrm{X}^{2} \Sigma_{\mathrm{g}}^{+}\right)-$ $\mathrm{Na}_{2}\left(2^{1} \Pi_{\mathrm{g}}\right)$ for these four groups of electrons, displayed in Figure $9 \mathrm{~b}$, shows that for each group a different range of internuclear distances is involved in the transition. However, they overlap and the summation leads to an $R$-independent transition probability. The conclusion from this is that there must be another ionization process responsible for the observed beat structure in the $\mathrm{Na}_{2}^{+}$ signal and different from direct photoionization.

(22) Engel, V. Chem. Phys. Lett. 1991, 178, 130. 


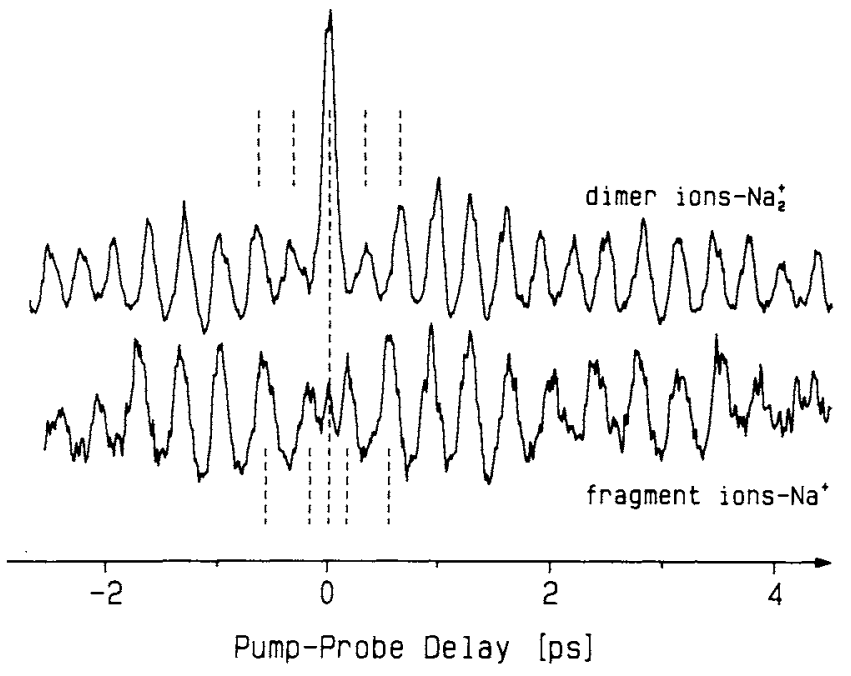

Figure 10. Comparison of transient $\mathrm{Na}_{2}^{+}$ionization spectrum (upper trace) with transient $\mathrm{Na}^{+}$photofragmentation spectrum (lower trace). Both spectra were measured under the same experimental conditions (85-fs pulse duration, $0.2-\mu \mathrm{J}$ pulse energy, $\lambda_{\max }=623 \mathrm{~nm}$ ). The modulation $\left(T \approx 309 \mathrm{fs}\right.$ ) in the $\mathrm{Na}_{2}^{+}$spectrum is in phase with the preparation of the wave packet at $t=0$, whereas the modulation $(T \approx 372 \mathrm{fs})$ in the $\mathrm{Na}^{+}$spectrum is $180^{\circ}$ out of phase.

In order to gain more insight into the anticipated ionization process, we have performed another time-resolved experiment where we now measured the variation of the "slow" $\mathrm{Na}^{+}$ionic fragment signal as a function of the delay time between the femtosecond pump and probe laser pulses. The experiment showed a striking result. The observed transient $\mathrm{Na}^{+}$photofragment signal is shown in the lower part of Figure 10, while in the upper part the transient molecular $\mathrm{Na}_{2}^{+}$ion signal is given for comparison. The oscillation period of the transient $\mathrm{Na}^{+}$fragmentation spectrum is obviously determined by the wave packet motion in the $2^{1} \Pi_{g}$ state. Moreover, it shows again the phase shift of about $180^{\circ}$ with respect to the zero delay time. The details of the $\mathrm{Na}^{+}$photofragmentation experiment and the analysis will be published. ${ }^{23}$

The results of this time-resolved photofragmentation experiment, however, strongly suggest that the "slow" $\mathrm{Na}^{+}$ionic fragments and those molecular $\mathrm{Na}_{2}^{+}$ions, which are formed via the outer turning point of the $2^{1} \Pi_{\mathrm{g}}$ state, have a common origin.

The excitation of the inner electron of the $\mathrm{Na}_{2}{ }^{*}(3 \mathrm{~s}, 3 \mathrm{~d})$ Rydberg state by the probe laser into a higher orbital $n^{\prime} l^{\prime}$, to form a neutral electronically doubly excited $\mathrm{Na}_{2}$ molecule, is such a process. A doubly excited molecule $\mathrm{Na}_{2}{ }^{*}\left(n l, n^{\prime} l\right)$ may electronically autoionize, to form $\mathrm{Na}_{2}^{+}$as well as the fragments $\mathrm{Na}^{+}+\mathrm{Na}+\mathrm{e}^{-}$ in a three-particle breakup, to give the observed electron and $\mathrm{Na}^{+}$ ionic fragment kinetic energy distributions shown in Figure 3a,b.

Reasons why such a "core" excitation occurs in this case only at the outer turning point of the $2^{1} \Pi_{\mathrm{g}}$ state could be the relative location of the two potentials involved and a strong $R$ dependence of their electronic transition moment. Note that no calculations have yet been reported for molecular doubly excited states in this energy range. ${ }^{24}$

The two-photon-pump and one-photon-probe ionization process, which involves excitation and decay of a doubly excited state, is illustrated in Figure 11. Except for the repulsive ${ }^{2} \Sigma_{u}^{+}$state ${ }^{25}$ and the estimated $\mathrm{Na}_{2}{ }^{* *}$ curve, all others are RKR potential curves. The pump laser prepares a wave packet at the inner turning point, which then propagates in about 180 fs to the outer turning point where the probe laser transfers the motion into the continuum by exciting a second electron forming doubly excited $\mathrm{Na}_{2}{ }^{* *}$

(23) Baumert, T.; Weiss, V.; Gerber, G. To be published.

(24) Only potential energy curves for excited states of $\mathrm{Na}_{2} * *$ up to the (3p +3 p) dissociation limit are reported by Henriet, A.; Masnou-Seeuws, F, J. Phys. B: At. Mol. Phys. 1987, 20, 671; J. Phys. B: At., Mol. Opt. Phys. 1990 23, 219 .

(25) Müller, W.; Meyer, W. J. Chem. Phys. 1984, 80, 3311. Schmidt, I. Thesis, Universitat Kaiserslautern, 1987 (unpublished).

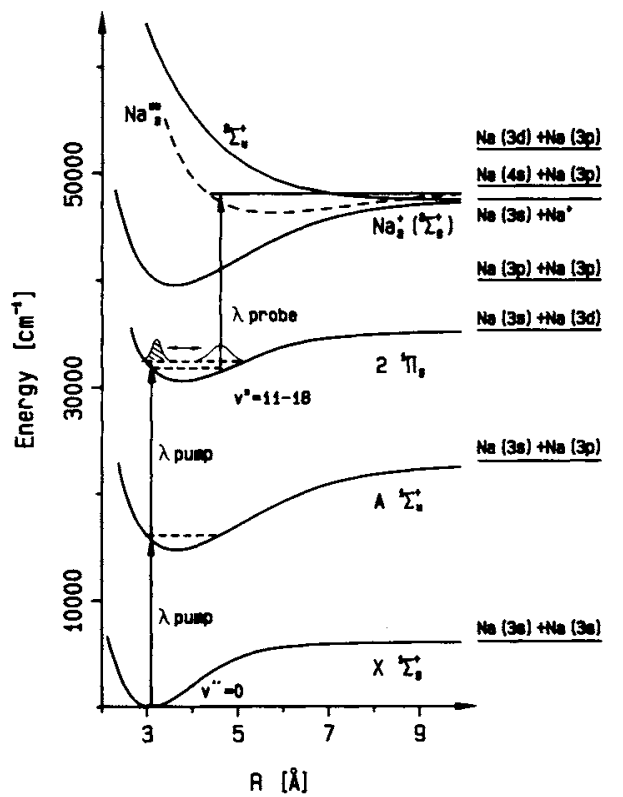

Figure 11. Potential curve diagram illustrating preparation of the wave packet at the inner turning point of the $2^{1} \Pi_{g}$ state and one-photon probe process leading to excitation of a bound doubly excited state of $\mathrm{Na}_{2}$. The probe process occurs only at the outer turning point.

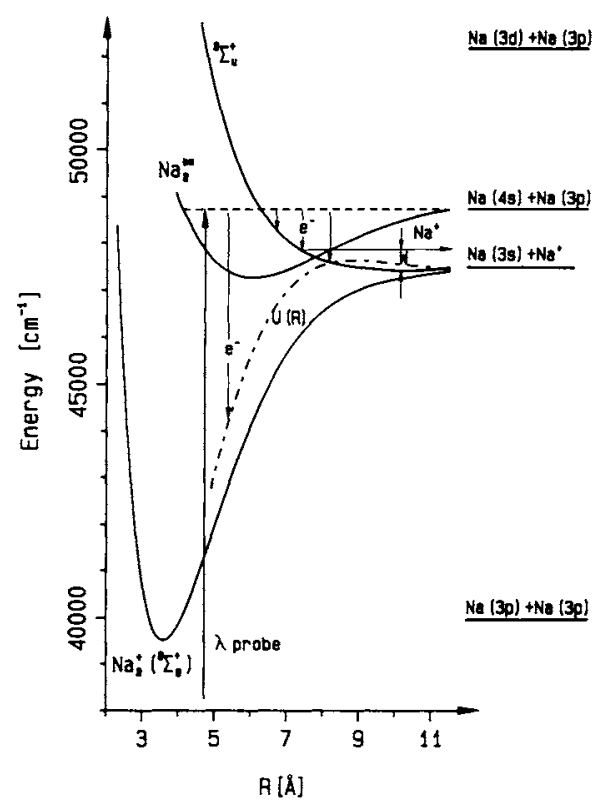

Figure 12. Excitation and decay processes of the doubly excited neutral diatomic sodium molecule. Electronic autoionization leads to $\mathrm{Na}^{+}+\mathrm{e}^{-}$ and autoionization-induced fragmentation in a three-particle breakup forms the fragments $\mathrm{Na}^{+}+\mathrm{Na}(3 \mathrm{~s})+\mathrm{e}^{-}$.

molecules. From the observed ion and electron spectra, these molecules have different ionization and fragmentation channels compared to direct photoionization and bound-free fragmentation. Figure 12, which is an enlargement of the upper part of Figure 11 , shows that doubly excited levels electronically autoionize into the molecular ion ground state $\mathrm{X}^{2} \Sigma_{\mathrm{g}}^{+}$. However, for internuclear distances greater than $6 \AA$, the vibronic levels of the doubly excited bound state interact additionally with the fragmentation continuum of the repulsive ${ }^{2} \Sigma_{u}^{+}$state of $\mathrm{Na}_{2}^{+}$. Therefore for larger $R$ a second channel is open for electronic autoionization now into the ${ }^{2} \Sigma_{u}^{+}$ ionization and fragmentation continuum.

Note that electronic autoionization transitions are not dipole transitions. Autoionization is a special case of an intramolecular perturbation that is governed by Kronig's selection rules. ${ }^{26}$ Because the parity of this doubly excited state is " $u$ ", the parity

(26) Kronig, R. deL. Z. Phys. 1928, 50, 347. 
of the final continuum state $\left(\mathrm{Na}_{2}^{+}+\mathrm{e}^{-}\right)$must also be " $u$ ". Thus, depending on the angular momentum of the outgoing electron, different final states of the molecular ion $\mathrm{Na}_{2}^{+}$with " $\mathrm{g}$ " and with " $u$ " parities can be formed in the electronic autoionization transitions.

This second autoionization decay leads to the observation of the "slow" ionic fragments $\mathrm{Na}^{+}$. The actual kinetic energy of the electron and the $\mathrm{Na}^{+}$fragment in this three-particle breakup is determined by the internuclear distance $R$ where the autoionization takes place. The energy released in this process is $E=E\left(\mathrm{Na}_{2}{ }^{* *}\right)$ $-E\left(\mathrm{Na}^{+}+\mathrm{Na}\right)$, and it is shared between the ejected electron and the ionic and neutral fragments $\mathrm{Na}^{+}$and $\mathrm{Na}$, respectively.

\section{Conclusion}

In conclusion, we report here on the first study in a molecular beam experiment where femtosecond pump-probe techniques have been used in combination with ion and photoelectron spectroscopy to study the dynamics of molecular multiphoton ionization. For the study we have chosen the spectroscopically well-known diatomic sodium molecule as a prototype. Our results reveal unexpected features of the dynamics of the absorption of many photons by a diatomic molecule. The measured pump-probe ionization and photofragmentation spectra show oscillatory structures and the Fourier analysis of the spectra indicate that under our experimental conditions two major contributions to the spectra exist. The detailed analysis of the transient $\mathrm{Na}_{2}^{+}$ionization spectra measured with 70 -fs pump and probe pulses shows that wave packet oscillations in the $A^{1} \Sigma_{u}^{+}$and the $2^{1} \Pi_{\mathrm{g}}$ potentials occur.

Three arguments led us to the conclusion that, for $\mathrm{Na}_{2}^{+}$, two different multiphoton ionization processes exist, requiring incoherent addition of the intensities to account for the measured signal: the observation of two different oscillation periods and their $180^{\circ}$ phase shift, the time structure of the transient "slow" $\mathrm{Na}^{+}$photofragmentation signal, and the difference potential analysis.
The direct photoionization of an excited electronic state, where one pump photon creates a wave packet in the $A^{1} \Sigma_{u}^{+}$state and two probe photons transfer that motion via the $2^{1} \Pi_{g}$ state into the $\mathrm{Na}_{2}^{+}\left({ }^{2} \Sigma_{\mathrm{g}}^{+}\right)$ionization continuum is one process. The second involves excitation of two electrons and subsequent electronic autoionization. Here two pump photons create a wave packet in the $2^{1} \Pi_{\mathrm{g}}$ state and one probe photon transfers its motion into the ionization continuum, but this happens only at the outer turning point of the $2^{1} \Pi_{\mathrm{g}}$ state. This particular process involves a bound doubly excited state of diatomic sodium, which through electronic autoionization forms $\mathrm{Na}_{2}^{+}$molecular ions and by autoionizationinduced fragmentation $\mathrm{Na}^{+}$ionic fragments. In this second multiphoton ionization process the probe photon is absorbed the first time about $180 \mathrm{fs}$ after the pump photons have been absorbed and then periodically after each round trip of the wave packet, but only at the outer turning point.

The time-resolved motion of the wave packets in two molecular potentials clearly shows there are two different multiphoton ionization processes rather than two intramolecular pathways.

An interesting problem, which will be addressed in a forthcoming publication, ${ }^{27}$ is the relative strengths of the two multiphoton ionization processes for higher and higher laser field intensities.

Acknowledgment. We thank Dr. V. Engel for many stimulating discussions, Drs. I. Gord and M. L. Polak for critical reading of the manuscript, and the Deutsche Forschungsgemeinschaft, SFB No. 276, "Korrelierte Dynamik hochangeregter atomarer und molekularer Systeme" for supporting this work. G.G. acknowledges a Visiting Fellowship, 1990-91 from the Joint Institute for Laboratory Astrophysics of the University of Colorado at Boulder.

Registry No. $\mathrm{Na}_{2}, 25681-79-2 ; \mathrm{Na}^{+}, 17341-25-2 ; \mathrm{Na}_{2}{ }^{+}, 12596-67-7$; $\mathrm{Na}_{3}{ }^{+}, 12767-53-2 ; \mathrm{Na}, 7440-23-5$.

(27) Baumert, T.; Engel, V.; Gerber, G. To be published. 